\title{
Cognitive priming: assessing the use of non-conscious perception to enhance learner's reasoning ability
}

\author{
Pierre Chalfoun, Claude Frasson \\ Université de Montréal, Dept. of Computer Science and Operations Research \\ 2920 chemin de la tour, H3T-1J8 QC, Canada \\ \{chalfoun, frasson\} @iro. umontreal.ca
}

\begin{abstract}
Current Intelligent Tutoring Systems (ITS) employ explicit and direct learning strategies when interacting with learners. Although these ITS use cognitive and logical models to analyze the conscious cognitive processes behind reasoning, we believe that in specific situations during knowledge acquisition, such as reasoning, unconscious cognitive processes are heavily solicited in the brain. In this paper, we will propose a complimentary and novel learning strategy to current ITS aimed at enhancing reasoning in a problem solving environment. This approach, called Cognitive Priming, is based on neural correlates of non-conscious perception. We will present two studies that have positively conditioned learners and enhanced different dimensions of their reasoning skills by employing a technique based on the science of subliminal perception. We will also present relevant cerebral data recorded throughout the studies and discuss the importance of such findings for the community.
\end{abstract}

Keywords: Cognitive priming, reasoning, problem solving, EEG, ITS.

\section{Introduction}

For more than twenty five years now, the aim of intelligent Tutoring Systems (ITS) has been to properly adapt learning sessions and material to the learner. Moreover, the availability, ease of use, and affordability of physiological devices have endowed current tutors with the ability to assess student's cognitive and affective states during learning [1]. Amongst the many important cognitive processes that occur during learning, properly assessing the reasoning ability of learners when acquiring knowledge is of paramount importance. Current ITS use explicit and direct learning strategies when interacting with learners and only assess conscious cognitive processes that occur during learning. However, it is now widely accepted that the unconscious mind does play a role in cognitive activity and in learning. Indeed, we believe, based on several experiments and well recorded phenomenon in recent neuroscience literature, that learning is a complex interplay between conscious and unconscious mechanisms in the brain and exploring and assessing these mechanisms is not only possible, but of great interest [2]. In general, this research is interested in exploring the domain of unconscious cognition and assess, using physiological sensors, the relevant cognitive mechanisms involved during reasoning in a problem solving envi- 
ronment. This paper, more specifically, will explore and assess the possibility of enhancing learner's reasoning ability in a problem solving environment by employing a technique based on neural correlates of non-conscious perception called "cognitive priming". The idea is to project answers to a problem slightly outside the learner's conscious awareness while active thinking is taking place thus increasing the reasoning process of learners. Contrary to popular belief, a large body of work in neuroscience has put forward strong evidence that learning simple to complex information can be done without perception or complete awareness at the task at hand [3, 4].

\section{Cognitive priming}

Before going further, we need to clearly establish the terminology that will be used in this paper. Unconscious cognition refers to the wide range of possible effects that unconscious mechanisms in the brain can have on cognitive processes such as learning and complex decision making. Non-conscious perception is the sub-branch of unconscious cognition that deals with all sensory-related stimuli that are processed unconsciously (e.g. images or sounds). Subliminal perception is a technique that transmits information without overloading the active cognitive channel by projecting a stimulus, called a prime, under the human conscious visual threshold. Masked priming is one of the most widely used technique for subliminal perception [5]. It consists in projecting for a very short time (20 to $40 \mathrm{~ms}$ ) a stimulus (such as a word or an image) preceded and/or followed by the projection of a mask (random figures or dashes) for a few hundred milliseconds. Cognitive priming is a special case of subliminal perception where the stimulus used (answer to a question for example) is aimed toward enhancing cognitive processes such as reasoning or decision making towards the goal of better knowledge acquisition. The essence of our work is inspired by a framework in the neuroscience of non-conscious perception and more specifically on to two landmark papers in Science and Nature where robust subliminal priming methodologies showed that that genuinely invisible primes could influence processing at a semantic level $[6,7]$. In light of all these findings, we have carefully designed subliminal primes in the form of images containing cognitively helpful information to the learner for a problem solving environment. We will now review some relevant work in areas close to our research interests before presenting our experiments.

\section{Related work}

To the best of our knowledge, we found no similar work in the ITS/AIED community that uses and assesses cognitive priming to attempt to enhance reasoning in a problem solving environment. The most relevant work however regarding our research has been done by Lowery and colleagues who demonstrated that subliminal primes can increase performance on midterm exams compared to neutral primes and that subliminal priming may have long-term effects on real-world behavior [8]. In HCI, one of the early works regarding subliminal cues for task-supported operation was the text editor program of Wallace [9] where Wallace and colleagues found that the frequency 
at which subjects demanded help was much lower when the required information was presented in subliminal matter. The Memory Glasses by DeVaul and colleagues [3] used wearable glasses that projects subliminal cues as a strategy for just-in time memory support. The objective was to investigate the effect of various subliminal cues (correct and misleading) on retention in a word-face learning paradigm and compare recall performance. Another use of priming for memory support can be found in the thesis of Shutte [10] where the author assessed the effects of brief subliminal primes on memory retention during an interference task. Although the results of these priming seemed very encouraging, the author cautions HCI designers that misusing subliminal priming that can lead to critical disruptions of ongoing tasks. After briefly reviewing the relevant work, we will now present the studies conducted where cognitive priming was used in two distinct experimental setups.

\section{Empirical studies conducted}

The learning task set in both of these experiments is to teach the construction of an odd magic square of any order with the use of 3 cumulative visual tricks (T1 to T3) requiring neither a calculator nor complex mental arithmetic operations. For a magic square of $5 \times 5$, the three tricks show how to properly fill the boxes in the square with numbers from 1 to 25 . Tricks are cumulative and a learner must use T1 to complete $\mathrm{T} 2$ and T2 to complete T3. Thus, T3 is more difficult to understand than T1 because it requires learners to have understood $\mathrm{T} 1$ and $\mathrm{T} 2$ respectively. (see [11] for details).

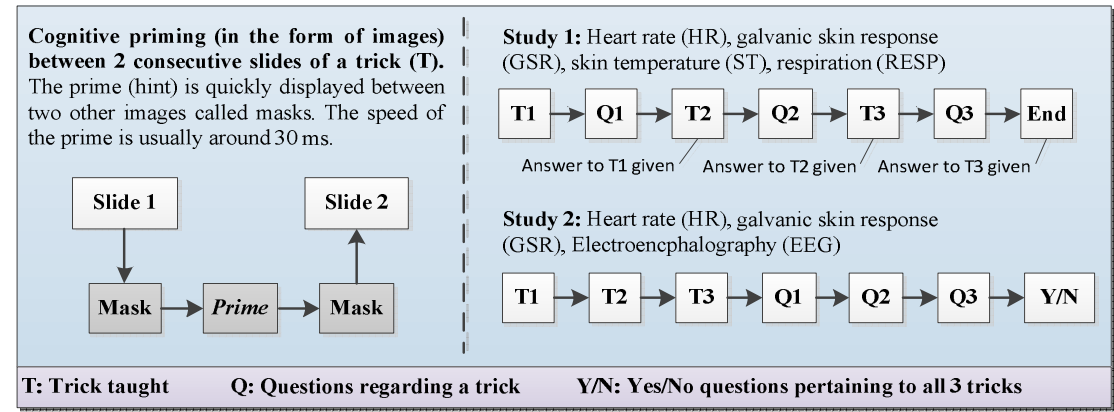

Fig. 1. Overall design for study 1 and 2.

The main objective of the studies was to learn the tricks whilst making the fewest possible mistakes. Learning performance metrics were our main criterions for evaluating learners' reasoning performance. The subliminal stimulus, thresholds, prime and masks were carefully chosen following the neural bases of subliminal priming [5] as well as accepted brain methodologies [12].

Study 1: using positive hints as cognitive primes. In this study, learners are shown multiple examples of each trick without explaining how the trick works. We present for each trick various Power Point slides of before and after states of the magic square 
for various numbers. Instead of giving away the answer, we ask the subjects to deduce the rule by themselves. The control group will try to deduce each trick without subliminal stimuli and conversely the condition group will be conditioned by a subliminal tutor that will prime learners. The primes are in the form of arrows pointing to the proper location on the square where the next number should appear. We will then compare performances, trick completion time and question completion time. Learning takes place in a 3D game-like environment called MOCAS. The interactions between the avatar's learner and the pedagogical agents are done via mouse clicks. The learners are instructed to continue once they are convinced they have discovered the inner working of each trick and cannot go back. They are asked a series of questions by virtual avatars related to the last trick learned. After answering all questions related to each learned trick, the answer to the current trick is revealed before learning the next trick. Physiological signals of the learners were also monitored in real-time and saved for off-line analysis. The signals were heart rate, galvanic skin response, respiration and skin temperature. A total of 30 participants were recruited for the experiment; they were assigned either to the experimental condition $(\mathrm{N}=15$; PositivePrime) or to the control condition ( $\mathrm{N}=15$; Control).

Results. Our hypothesis in this study was that cognitive primes were to have positive effects on reasoning, and consequently on performance and mistakes made. The results obtained show that PositivePrime's overall performance was statistically different $(\mathrm{p}=.023$, alpha $=0.05)$ than the control group and 2.7 times more efficient on average (44\% less mistakes overall with the presence of the subliminal module). Furthermore, subliminal priming at specific intervals seem to significantly reduce the time spend on each question. Indeed, time spent on each question by primed learners is reduced by an overall factor of 1.3 (Single factor ANOVA $p=.023$, alpha $=0.05$ ). It is important to note that NO subliminal priming is done during the questions. All the priming is done during the tricks taught. The answer to the questions is not projected subliminally when the question is asked. We believe these encouraging results can be explained by the fact that the subliminal primes are goal-relevant to the cognitive task at hand and might have acted as a "catalyst" for quickly converging to a solution as observed by previous studies [13]. However, we wanted to verify the validity of this priming strategy by conducting a second study where we introduced primes (called miscues) that were designed to throw off learners in order to compare results with positive primes used here.

Study 2: using positive and misleading hints as cognitive primes. In this study, we are teaching the same lesson (learning how to construct a magic square in 3 simple tricks) but within a 2D system that looks very similar to an online exam session. Although learners still had to infer their own solutions and correctly figure out the algorithm used in each trick, the solution to each trick was never presented (see fig. 1). Thus, each learner had to induce the rules and construct a mental model of the overall solution. Furthermore, learners reported how they figured each trick by choosing between the following: I deduced the trick by intuition, logic, a little of both (variable Trick answer type). A fixed time limit of 45 seconds for the questions was imposed. Failing to give an answer within the allowed time was considered a mistake. Learners 
also reported how they answered each question by choosing between the following: I answered the question by guessing the answer, by intuition or by logical deduction (variable Question answer type). After giving their answer, a green check or a red cross appears for 2 seconds indicating to the learner if they made a correct or wrong choice respectively. The main intent for these changes is to associate relevant brains states with how learners reasoned and resolved problems. Physiological signals of the learners were also monitored in real-time and saved for off-line analysis. The signals were EEG (brainwaves), heart rate and galvanic skin response. A total of 43 participants were recruited for the experiment; they were assigned either to the answer group ( $\mathrm{N}=14$; Answer_cues), to the misleading group ( $\mathrm{N}=14$; Miscue) or to the control group $(\mathrm{N}=15$; Control). Each learner was compensated with $10 \$$.

Results. Our hypothesis in this study was that cognitive positive primes only, and not miscues, were to have positive effects on reasoning, and consequently on performance and mistakes made. We examined results related to performance (number of mistakes) with regards to the way learning occurred (Trick answer type), the way learners answered questions (Question answer type) and the group (Answer_cues, Miscue, Control). Significant effects from a four way cross-tabulation analysis were only found for the variables Trick answer type* group with regards to the number of mistakes with the following combinations: Logic*Answer_cues $(\mathrm{p}=.002$, alpha $=0.05$, chi-square $=16.949)$, A little of both*Answer_cues $(\mathrm{p}=.048$, alpha $=0.05$, chi-square = 9.117). Results seem to indicate that only Answer_cues, and not miscues, do significantly influence logical reasoning and decision making when learning a trick logically. From the EEG data we were interested in investigating changes in two metrics that have previously been reported as relevant indicators of insightful problem solving (40Hz right asymmetry) [14] and complex arithmetic processing (Beta2 left asymmetry) [15]. We observed that the asymmetry values for the $40 \mathrm{~Hz}(\mathrm{p}=.003$, alpha $=$ $0.05)$ and $\operatorname{Beta} 2(\mathrm{p}=.04$, alpha $=0.05)$ in the Answer_cues group are significantly different than the Miscue group for the third and most difficult trick. The Answer_cues group seems to shift their attention from a complex arithmetic process (Beta2 left asymetry decrease) toward an "insightful" problem solving strategy $(40 \mathrm{~Hz}$ right asymetry increase), thus involving the right side of the brain, known to be an important actor in insightful problem solving. The combination of these two metrics could indeed be an interesting indicator of a change in the reasoning stategy from complex arithmetics to insightful reasoning during problem-solving.

\section{Conclusion}

We have presented in this paper a novel approach to enhance reasoning and learning in a problem solving environment with cognitive priming. This technique aims at enhancing unconscious processes involved in learning, namely reasoning, by projecting information under the visual threshold of learners without neither overloading the current cognitive channel nor disturbing the learning session. Furthermore, cerebral recording have shown that it might be possible to assess not only classical reasoning 
but also intuitive reasoning. Expected benefits from this technique are two-fold. First, the reasoning ability of learners is strengthened by the added information outside of conscious awareness. Second, the supplementary cognitive data does not hamper or interrupt active cognitive processes. Many interesting challenges remain however for future work such as ethical aspects and usage of this technique in a more complex scenario where deep learning might occur. We are currently working on all these issues and hope to present relevant findings to the community in the near future.

\section{References}

1. Nkambou, R., J. Bourdeau and R. Mizoguchi, eds. Advances in Intelligent Tutoring Systems. Studies in Computational Intelligence. Vol. 308. 2010, Springer. 508.

2. Hassin, R.R., J.S. Uleman and J.A. Bargh, The new unconsciousness2005: Oxford University Press, New York. 575.

3. DeVaul, R.W., A. Pentland and V.R. Corey, The Memory Glasses: Subliminal vs. Overt Memory Support with Imperfect Information, in IEEE International Symposium on Wearable Computers2003, IEEE Computer Society: New York. p. 146-153.

4. Nunez, J.P. and F.D. Vincente, Unconscious learning. Conditioning to subliminal visual stimuli. The Spanish Journal of Psychology, 2004. 7(1): p. 15.

5. Del Cul, A., S. Baillet and S. Dehaene, Brain Dynamics Underlying the Nonlinear Threshold for Access to Consciousness. PLoS Biololgy, 2007. 5(10): p. 2408-2423.

6. Dehaene, S., L. Naccache, G. Le Clec'H, E. Koechlin, M. Mueller, G. Dehaene-Lambertz, P.F. van de Moortele and D. Le Bihan, Imaging unconscious semantic priming. Nature, 1998. 395: p. 597-600.

7. Greenwald, A.D., S.C. Draine and R.L. Abrams, Three cognitive markers of unconscious semantic activation. Science, 1996. 273: p. 1699-1702.

8. Lowery, B.S., N.I. Eisenberger, C.D. Hardin and S. Sinclair, Long-term effect of subliminal priming on academic performance. Basic and applied social psychology, 2007. 29(2): p. 151-157.

9. Wallace, F.L., J.M. Flaherty and G.A. Knezek, The Effect of Subliminal HELP Presentations on Learning a Text Editor. Information Processing and Management, 1991. 27(2/3): p. 7.

10. Schutte, P.C., Assessing the Effects of Momentary Priming on Memory Retention During an Interference Task, in Computer Science2005, Virginia Commonwealth University: Virginia. p. 103.

11. Chalfoun, P. and C. Frasson, Subliminal cues while teaching: HCI technique for enhanced learning. Advances in Human Computer Interaction : Special Issue on Subliminal Communication in HumanComputer Interaction, 2011. 2011(1).

12. Pessiglione, M., P. Petrovic, J. Daunizeau, S. Palminteri, R.J. Dolan and C.D. Frith, Subliminal Instrumental Conditioning Demonstrated in the Human Brain. Neuron, 2008. 59: p. 561-567.

13. Strahan, E.J., S.J. Spencer and M.P. Zanna, Subliminal priming and persuasion: Striking while the iron is hot. Journal of Experimental Social Psychology, 2002. 6(38): p. 13.

14. Sandkühler, S. and J. Bhattacharya, Deconstructing insight: EEG correlates of Insightful Problem Solving. PLOS One, 2008. 3(1).

15. Hyungkyu, K., C. Jangsik and L. Eunjung, EEG Asymmetry Analysis of the Left and Right Brain Activities During Simple versus Complex Arithmetic Learning. Journal of Neurotherapy, 2009. 13. 